\title{
Generic Versus Disease-specific Measures of Health-related Quality of Life in Systemic Lupus Erythematosus
}

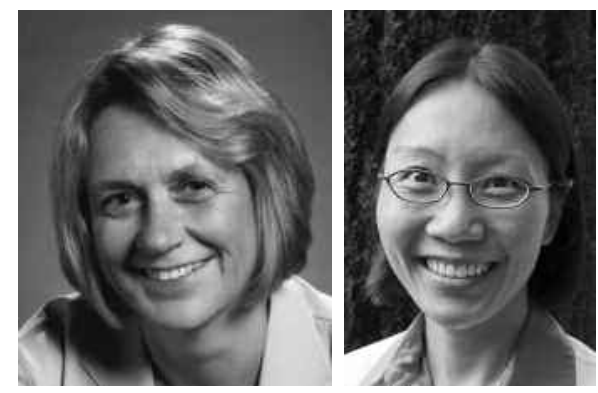

Systemic lupus erythematosus (SLE) is an autoimmune inflammatory disease that significantly affects health-related quality of life (HRQOL): physical, psychologic, mental, and social aspects of well-being that are influenced by disease, in the context of life experiences and expectations specific to each patient ${ }^{1}$. A relapsing, remitting chronic disease, SLE results in disability in 20\%-40\% of afflicted young and middle-aged women and $\mathrm{men}^{2}$. In patients with SLE, HRQOL is influenced by disease activity and symptoms of fatigue, depression, pain, sleep disturbances, and cognitive dysfunction ${ }^{3}$. Across 5 randomized controlled trials (RCT) in SLE, baseline HRQOL scores were low and were similar to those of subjects following myocardial infarction or with chronic congestive heart failure ${ }^{4}$. Lower scores were highly correlated with history of renal disease, presence of anti-dsDNA antibodies, higher disease activity scores by Systemic Lupus Erythematosus Disease Activity Index (SLEDAI) and/or Safety of Estrogens in Lupus Erythematosus National Assessment (SELENA-SLEDAI), hypocomplementemia, African American descent, and age. A variety of therapeutic interventions, including pharmacologic and biologic therapies, have been shown in RCT to improve HRQOL, including prasterone, mycophenolate mofetil, abetimus sodium, oral contraceptive, and hormone replacement therapy in the SELENA trials, as well as monoclonal antibodies epratuzumab and belimumab ${ }^{5,6,7,8}$.

In 1998, an international consensus conference on outcome measures in rheumatology (OMERACT 4) recommended that 4 core domains be assessed in RCT and longitudinal observational studies (LOS) in SLE: disease activity, HRQOL, adverse events, and damage ${ }^{9}$. OMERACT has also recommended that both generic and disease-specific instruments be utilized to measure HRQOL. Ongoing efforts to develop promising therapies for SLE have demonstrated the importance of including patient-reported outcomes (PRO) to assess HRQOL in RCT.

Measurement of HRQOL adds a unique dimension to the assessment of treatment response. It has been shown that HRQOL may be influenced by, but does not correlate highly with, disease activity and damage measures, and thus offers a different domain of assessment ${ }^{10}$. A widely used generic measure, the Medical Outcomes Survey Short-Form 36 (SF-36), has to date best revealed the effect of SLE on HRQOL in RCT and LOS. SF-36 has been well validated in SLE, translated into many languages, and cross-culturally validated, and has frequently been utilized as the only PRO measure in $\mathrm{RCT}^{5,6,7,8,9}$. It comprises 8 domains: physical functioning, role physical, bodily pain, general health perceptions, vitality (which includes fatigue, energy, and "pep"), social functioning, role emotional, and mental health effect; these are combined into physical and mental component summary scores (PCS and MCS, respectively). The summary scores are not fully independent of each other, and examining changes across all 8 domains reveals a more complete picture of the impact of disease as well as treatment-associated changes.

Several disease-specific instruments have been designed to assess HRQOL in SLE: LupusQoL, L-QoL, SLE-QoL, and Lupus-PRO. Derived from semistructured interviews with SLE patients, the LupusQoL questionnaire contains 34 items across 8 domains: Physical Health, Emotional Health, Body Image, Pain, Planning, Fatigue, Intimate Relationships, and Burden to Others ${ }^{11}$. It emphasizes areas such as sleep, body image, and sexual health, which are not specifically queried in SF-36. LupusQoL has demonstrated good internal consistency, test-retest reliability, and concurrent validity with the generic SF-36 ${ }^{12}$. Developed in the UK, it has now been adapted and validated for use in the US and Canada, and a Spanish version has been validated ${ }^{13,14}$. The L-QoL was developed from patient interviews and validated in the UK, and is based on the concept that improvements in HRQOL derive from the ability and capacity of individuals to satisfy their needs ${ }^{15}$. The Systemic Lupus Erythematosus specific Quality of Life (SLEQOL), devel-

See Is there an advantage over SF-36 with a QOL measure specific to SLE?, page 1898 
oped in Singapore, contains 40 items selected by 100 patients from 51 items suggested by rheumatologists, but lacks a formal qualitative study involving patient input an acknowledged limitation ${ }^{16,17}$. It has been validated in both English and Chinese versions. The lupus-specific PRO measure Lupus-PRO, developed in the US, includes 10 domains of HRQOL as well as 4 that are considered to reflect "non-HRQOL" including desires/goals, coping, social support, and medical care ${ }^{18}$. The summary HRQOL score correlated well with health utility measures SF-6D, derived from SF-36, and EuroQol EQ-5D. Undergoing further validation, it is also being utilized to develop a patientreported instrument for use in day-to-day practice: Lupus Tracker ${ }^{19,20}$. One challenge with these disease-specific instruments is that most are culturally and geographically distinct, validated in the UK, Singapore and the US, and do not readily lend themselves to use in multinational trials required in SLE. This is exemplified by a recent focus group study conducted by Thumboo and colleagues in Singapore among English-speaking Asian patients with SLE ${ }^{18}$. They identified 4 domains not included in existing SLE-specific measures of HRQOL: disease impact upon family, relationships, freedom, and stigma and discrimination - where importance of the latter may be accentuated in the Asian sociocultural context.

In this issue of The Journal, Touma, et al compare the generic SF-36 to the disease-specific LupusQoL in a cohort of 41 patients, assessed monthly for a total of 376 patient visits ${ }^{21}$. Forty-one were "baseline" visits, 127 visits demonstrated remission in 23 patients, 14 demonstrated flare in 10 patients, 11 showed improvement in 8 patients, and 183 visits demonstrated no change in 34 patients. The objectives of this investigation were to determine whether the LupusQoL questionnaire contributed additional information not obtained using SF-36 in this SLE cohort and to evaluate its responsiveness to changes in disease activity. Scores across comparable and noncomparable domains of SF-36 and LupusQoL were assessed by effect sizes and standardized response means. Interestingly, the study demonstrated strong correlations between comparable domains of LupusQoL and SF-36. And for the 4 noncomparable domains of LupusQoL, correlations existed between each domain and at least one component score of SF-36: Body Image and SF-36 MCS; Planning and SF-36 MCS; Intimate Relationships and SF-36 PCS; and Burden to Others and SF-36 MCS. Both SF-36 and LupusQoL were responsive to clinically significant changes in disease activity in this patient population with relatively low disease activity, with median SLEDAI-2K scores of 2 . The authors conclude that these 2 instruments were equivalent in assessing HRQOL over time in SLE, and offered complementary information. LupusQol has been validated in US patients with higher disease activity with median SLEDAI-2K scores of $4^{19}$. Work is under way to similarly compare these 2 instruments in patients with moderate to severe SLE disease activity 22 .

The challenges of conducting trials in SLE are well recognized, and published. HRQOL is an important domain for assessment in SLE. Disease activity, damage, and HRQOL are independent of one another, reflecting different domains affected by SLE, and all should be assessed in a patient with SLE to clarify the complete clinical picture; moreover, they add discriminative power when assessing promising new therapies. Use of different disease-specific instruments to assess HRQOL limits comparison across studies, hence the increasing use of SF-36 is encouraging. As HRQOL is a widely utilized generic measure validated in a variety of rheumatic diseases (including rheumatoid arthritis, osteoarthritis, psoriatic arthritis, gout, fibromyalgia, and systemic sclerosis), its use enables comparisons between these diseases as well as to normative data ${ }^{7,8}$. Further, HRQOL appears to discriminate between SLE disease activity and fibromyalgia ${ }^{8}$. Recently it was demonstrated that changes in SF-36 summary and domain scores, in particular those related to mental health, were strongly associated with clinical outcome of neuropsychiatric events in patients with SLE, underscoring its importance in this regard ${ }^{23}$.

SF-36 may also be utilized to derive health utility scores, such as the SF-6D, which permit economic analyses of cost of disease and treatment and facilitate comparisons across disease states ${ }^{8}$. This is important, as the frequently used EQ-5D is less sensitive to change than SF-36 in many rheumatic diseases, summarizing health status using only 5 questions ${ }^{24}$.

Recent data demonstrate the value of utilizing not only a generic measure, such as SF-36, but also a disease-specific measure. Now that LupusQoL has been adapted and validated for use in the US and Canada and a Spanish version validated, there is an opportunity to use a more broadly applicable disease-specific instrument to assess HRQOL. Another option, illustrated by Thumboo, et al, would be to adapt the PROMIS (Patient-Reported Outcomes Measurement Information System) item banks for use as diseasespecific measures of HRQOL in SLE, which may better accommodate cross-cultural differences.

In summary, patient-reported HRQOL, an important domain to be assessed in SLE studies, provides unique information that cannot be obtained from measures of disease activity and/or damage. This is best measured by use of both generic and well validated disease-specific instruments.

VIBEKE STRAND, MD, FACP, FACR,

Clinical Professor, Adjunct, Division of Immunology/Rheumatology, Stanford University;

ALVINA D. CHU, MD,

Adjunct Clinical Instructor, Division of Immunology/Rheumatology, Stanford University School of Medicine, Stanford, California, USA

Address correspondence to Dr. Strand; E-mail: vstrand@stanford.edu Personal non-commercial use only. The Journal of Rheumatology Copyright @ 2011 . All rights reserved. 


\section{REFERENCES}

1. Strand V, Russell AS. Workshop report: WHO/ILAR Taskforce on Quality of Life. J Rheumatol 1997;24:1630-3.

2. Baker K, Pope J. Employment and work disability in systemic lupus erythematosus: a systematic review. Rheumatology 2009; 48:281-4.

3. Kiani AN, Petri M. Quality-of-life measurements versus disease activity in systemic lupus erythematosus. Curr Rheumatol Rep 2010;12:250-8.

4. Thumboo J, Strand V. Health-related quality of life in patients with systemic lupus erythematosus: an update. Ann Acad Med Singapore 2007;36:115-22.

5. Strand V, Aranow C, Cardiel MH, Alarcon-Segovia D, Furie R, Sherrer Y, et al. Improvement in health-related quality of life in systemic lupus erythematosus patients enrolled in a randomized clinical trial comparing LJP 394 treatment with placebo. Lupus 2003;12:677-86.

6. Strand V, Crawford B. Improvement in health-related quality of life in patients with SLE following sustained reductions in anti-dsDNA antibodies. Expert Rev Pharmacoecon Outcomes Res 2005;5:317-26.

7. Strand V, Crawford B, Singh J, Choy E, Smolen JS, Khanna D. Use of "spydergrams" to present and interpret SF-36 health-related quality of life data across rheumatic diseases. Ann Rheum Dis 2009;68:1800-4.

8. Strand V, Levy RA, Cervera R, Petri M, Rudge H, Pineda L, et al. for the BLISS-52 and -76 Study groups. Belimumab, a BlyS-specific inhibitor, improved fatigue and SF-36 physical and mental component summary scores in patients with SLE: BLISS-52 and -76 Studies [abstract]. Arthritis Rheum 2010;62 Suppl:S773.

9. Strand V, Gladman D, Isenberg D, Petri M, Smolen JS, Tugwell P. Endpoints: consensus recommendations from OMERACT IV. Outcome Measures in Rheumatology. Lupus 2000;9:322-7.

10. Gladman D, Urowitz MB, Ong A, Gough J, MacKinnon A. Lack of correlation among the 3 outcomes describing SLE: disease activity, damage and quality of life. Clin Exp Rheumatol 1996;14:305-8

11. McElhone K, Abbott J, Shelmerdine J, Bruce IN, Ahmad Y, Gordon C, et al. Development and validation of a disease-specific health-related quality of life measure, the LupusQol, for adults with systemic lupus erythematosus. Arthritis Rheum 2007; 57:972-9.

12. McElhone K, Castelino M, Abbott J, Bruce IN, Ahmad Y, Shelmerdine J, et al. The LupusQoL and associations with demographics and clinical measurements in patients with systemic lupus erythematosus. J Rheumatol 2010;37:2273-9.

13. Jolly M, Pickard AS, Wilke C, Mikolaitis RA, Teh LS, McElhone $\mathrm{K}$, et al. Lupus-specific health outcome measure for US patients: the LupusQoL-US version. Ann Rheum Dis 2010;69:29-33.
14. Gonzalez-Rodriguez V, Peralta-Ramirez MI, Navarrete-Navarrete N, Callejas-Rubio JL, Santos Ruiz AM, Khamashta M. Adaptation and validation of the Spanish version of a disease specific quality of life measure in patients with systemic lupus erythematosus: the Lupus quality of life. Med Clin (Barc) 2010;134:13-6.

15. Doward LC, McKenna SP, Whalley D, Tennant A, Griffiths B, Emery P, et al. The development of the L-QoL: a quality-of-life instrument specific to systemic lupus erythematosus. Ann Rheum Dis 2009;68:196-200.

16. Leong KP, Kong KO, Thong BY, Koh ET, Lian TY, Teh CL, et al. Development and preliminary validation of a systemic lupus erythematosus-specific quality-of-life instrument (SLEQOL). Rheumatology 2005;44:1267-76.

17. Ow YLM, Thumboo J, Cella D, Cheung YB, Fong KY, Wee HL. Domains of health related quality of life important and relevant to multiethnic English speaking Asian SLE patients: A focus group study. Arthritis Care Res 2011;63:899-908.

18. Jolly M, Pickard AD, Fogg L, Wilke CT, Lin HQ, Mikolaitis RA, et al. US Patient Derived Lupus-Specific Patient Reported Outcome Measure (Lupus-PRO ${ }^{\odot}$ ): Validity and reliability [abstract]. Arthritis Rheum 2008;58 Suppl:S805.

19. Jolly M, Kosinski M, Garris CP, Jhingran PM, Mikolaitis RA, Dennis G, et al. Development of the Lupus Impact Tracker ${ }^{\mathrm{TM}}$ *: A tool for patients and HCPs to assess and monitor the impact of systemic lupus erythematosus (SLE) [abstract]. Ann Rheum Dis 2011;70 Suppl 3:501.

20. Jolly M, Kosinski M, Garris CP, Jhingran PM, Mikolaitis RA, Dennis G, et al. Validation of the Lupus Impact $\operatorname{Tracker}^{\mathrm{TM}} *$ : A tool for patients and HCPs to assess and monitor the impact of systemic lupus erythematosus (SLE) [abstract]. Ann Rheum Dis 2011;70 Suppl 3:425.

21. Touma Z, Gladman DD, Ibañez D, Urowitz MB. Is there an advantage over SF-36 with a quality of life measure that is specific to systemic lupus erythematosus? J Rheumatol 2011;38:1898-905.

22. Touma Z, Urowitz MB, Taghavi-Zadeh S, Gladman DD. Comparison of Lupus Quality of Life and SF-36 questionnaires in lupus patients with moderate to severe disease activity [abstract]. Ann Rheum Ds 2011;70 Suppl 3:698.

23. Hanly JG, Urowitz MB, Jackson D, Bae SC, Gordon C, Wallace DJ, et al. SF-36 summary and subscale scores are reliable outcomes of neuropsychiatric events in SLE. Ann Rheum Dis 2011;70:961-7

24. Wolfe F, Michaud K, Li T, Katz RT. EQ-5D and SF-36 quality of life measures in systemic lupus erythematosus: comparisons with rheumatoid arthritis, noninflammatory rheumatic disorders, and fibromyalgia. J Rheumatol 2010;37:296-304

J Rheumatol 2011;38:1821-3; doi:10.3899/jrheum.110766 